\title{
A survey of cultural competence of critical care nurses in KwaZulu-Natal
}

\author{
J de Beer,' PhD; J Chipps, ${ }^{2} \mathrm{PhD}$ \\ ' School of Nursing and Public Health, University of KwaZulu-Natal, Durban, South Africa and College of Nursing- Jeddah, King Saud bin \\ Abdul Aziz University for Health Sciences, Saudi Arabia \\ 2 School of Nursing, University of the Western Cape, Cape Town, South Africa
}

Corresponding author: J de Beer (debeerj@ukzn.ac.za)

Background. Nurses are primary caregivers and have a key role in providing care in a culturally diverse healthcare system, such as in South Africa (SA). Nurses need cultural competence in the management of patients within this cultural context. A healthcare system staffed by a culturally competent workforce can provide high-quality care to diverse population groups, contributing to the elimination of health disparities.

Objective. To describe the self-rated levels of cultural competence of nurses working in critical care settings in a selected public hospital in SA.

Methods. A quantitative descriptive survey was conducted with nurses from eight critical care units in a public hospital in KwaZulu-Natal Province, using the Inventory to Access the Process of Cultural Competency - Revised (IAPCC-R) cultural competence questionnaire.

Results. The overall cultural competence score for the respondents was 70.2 (standard deviation 7.2) out of a possible 100, with 77 (74\%) of the respondents scoring in the awareness range, $26(25 \%)$ in the competent range, and only 1 in the proficient range. Nurses from non-English-speaking backgrounds scored significantly higher in cultural competence than English-speaking nurses.

Conclusion. In addressing the many faces of cultural diversity, healthcare professionals must realise that these faces share a common vision: to obtain quality healthcare services that are culturally responsive and culturally relevant to the specific cultural group.

S Afr J Crit Care 2014;30(2):50-54. DOI:10.7196/SAJCC.188

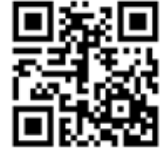

The growing interest in culture and healthcare dates back to the days of Florence Nightingale, who touched on the concept of transcultural nursing in the 19th century when advising British nurses working in India to take into account the cultural background of their patients. In the 1950s, transcultural nursing was introduced as a formal area of study and practice for nurses striving towards providing optimal and culturally appropriate care for patients. ${ }^{[1]}$
Everyone has a culture, and to provide culturally appropriate care, a nurses must understand their own culture and that of the nursing profession. Values such as caring, empathy, truthfulness, promoting health and autonomy, and respecting patients' choices influence how a nurse interacts with patients. ${ }^{[2]}$ Caring for patients from diverse backgrounds is a daily reality for nurses who are expected to provide both clinically safe and culturally sensitive care. ${ }^{[3]}$ Hence, healthcare professionals are faced with the challenge of providing culturally competent healthcare. 
Cultural competence refers to the knowledge and skills nurses should possess in order to care for patients from a cultural background different from their own. ${ }^{[4]}$ Cultural competence in nursing refers to the ability to take into account people's cultural beliefs, behaviours and needs in order to provide efficient healthcare, and is not merely a skill but a process. ${ }^{[5]}$ Cultural competence begins with an awareness of one's own cultural beliefs and practices, with the recognition that people from other cultures may not share these. To provide culturally competent care, healthcare professionals must be willing and able to provide family- and patient-centred care by adjusting their attitudes and behaviours to the needs of diverse patient groups. ${ }^{[6]}$

Healthcare professionals who lack cultural competence may be putting patients at risk for delays in treatment, inappropriate diagnosis, non-compliance with healthcare regimens, and even death of patients. Although healthcare professionals may not see themselves as overtly racist or neglectful, they could be missing pertinent healthcare findings due to 'cultural blindness. ${ }^{[7]}$ A culturally competent nurse should assess each patient individually and not make assumptions about a patient's beliefs and health practices. In addition, a culturally competent nurse must develop cultural sensitivity that involves being aware and utilising knowledge relating to culture, gender or sexual orientation. ${ }^{[8]}$ Andrews and Boyle ${ }^{[9]}$ stated that people subconsciously tend to view other people by using their own customs as standards for judgement, with low levels of knowledge of other cultures and an unquestioning belief in the superiority of one's own culture.

Cultural competence can be achieved in nurse education by developing a taxonomy that includes five areas: cultural awareness, cultural knowledge, cultural understanding, cultural sensitivity and cultural competence. ${ }^{[8]}$ Wittig $^{[10]}$ stated that nursing students will be able to identify some beliefs and practices used to care for individuals of a different culture if nursing programmes are teaching and implementing culturally competent care practices in their curriculum. Nursing students need to learn cultural competency concerning the population they work with, and although a knowledge of all cultural groups is not practical, students should learn to appreciate diversity, avoid prejudice and provide culturally sensitive care to patients.

Medical and nursing practice has two components: firstly, it involves scientific skills in physiology, pharmacology and surgery; and secondly, it involves social and cultural skills. Patients should not be viewed as mobile biochemical assemblies. Yet, one of the most striking aspects of modern medicine is not just its rapidly increasing technological sophistication, but the fact that healthcare professionals have come to evaluate themselves, and define their activities, primarily in technical terms. Everyday healthcare comprises both social and technical procedures. If healthcare professionals' social and cultural skills do not match their technical skills, their therapeutic effectiveness will be impaired. ${ }^{[1]}$

Critical care nurses (CCNs) are often advocates and executors of patient care in the critical care setting, a setting which is usually characterised as a technological advancement. It is in this clinical setting that traditional practices provide some sense of hope and comfort for both the patient and family, therefore planned care must be congruent with the patient's values and belief system. South Africa (SA) has diverse traditions and practices. The goal of $\mathrm{CCNs}$ is to provide the best possible care for their patients; therefore CCNs need to understand the cultural differences that may cause conflict, potentially compromising outcomes, and should familiarise themselves with cultural traditions specific to the SA population. Flowers ${ }^{[12]}$ noted with concern that during resuscitative attempts on patients, family members are often excluded even though certain cultures have a strong sense of family. Lack of cultural awareness and inability to provide culturally competent care can lead to conflict, increased levels of anxiety, and stress among nurses, patients and patients' relatives.

This study focused specifically on the practices of CCNs in the province of KwaZulu-Natal, where cultural diversity demands healthcare professionals to take cognizance of a patient's traditional beliefs on illness and healing.

\section{Models of cultural competence}

According to Leininger and McFarland ${ }^{[13]}$ care and culture are inextricably intertwined, and transcultural nursing focuses on the understanding of health-illness practices, beliefs and values from within patients' cultural groups. According to Leininger, ${ }^{[14]}{ }^{k n o w-}$ ledge of meanings and practices of diverse cultures is vital to guide nursing decisions and actions in providing culturally congruent care. Care is seen as the essence and central dominant unifying focus of nursing. Cultural care would provide a distinctive feature by which to know, interpret and explain nursing as a discipline and profession. It is important to provide knowledge on transcultural constructs and practices in order to provide culturally congruent care to people of different or similar cultures, so as to maintain or regain their wellbeing and health, or help them to face death in a culturally appropriate way.

Models of cultural competency (Andrews and Boyles, 2002; Campinha-Bacote, 1999, 2002, 2005, 2008; Leininger, 1982, 2002; Purnell, 2002; Ramsden, 1992) have been criticised for referring to cultural groups in terms of ethnicity, resulting in a 'narrow, essentialist and limiting' view of culture, as opposed to the more fluid constructionist view. Constructionists define patients and clients as the 'other' in opposition to the 'non-other' society and caregiver. ${ }^{[15]}$ This is evident in Leininger's theory of cultural care diversity and universality in caring for the culturally different, which epitomises this discourse. ${ }^{[15]}$

The Culturally Competent Model of Care designed by CampinhaBacote $^{[4]}$ is a cultural competence model that incorporates elements from all of the above models. The model comprises five components of a nurse-patient relationship in a cultural context (Table 1). The Inventory for Assessing the Process of Cultural Competence - Revised (IAPCC-R) is a self-assessment tool developed in 2002 to measure cultural competence in healthcare delivery using the Culturally Competent Model of Care as the framework. ${ }^{[4]}$ The tool has been used to assess cultural competence in a variety of healthcare professionals, and has been studied in the USA and internationally with internal consistency reliability statistics ranging from 0.65 to $0.9 .^{[16]}$

\section{Objective}

To describe the self-rated levels of cultural competence of CCNs working in critical care units (CCUs) in a public hospital in SA.

\section{Methods}

A quantitative survey of 168 registered nurses working in eight CCUs in a public hospital in KwaZulu-Natal was conducted using a questionnaire and the IAPCC-R tool to measure self-rated cultural competence. Stratified quota sampling from each CCU at the selected hospital was conducted. 
Table 1. Component of culturally competent care ${ }^{[4]}$

\begin{tabular}{ll}
\hline Cultural construct & Definition \\
\hline Cultural desire & The nurse must be motivated to become involved in the process of becoming culturally competent. \\
Cultural awareness & $\begin{array}{l}\text { The nurse becomes sensitive to the values, beliefs, lifestyles and practices of the patient and identifies } \\
\text { his/her own values, biases and prejudices. }\end{array}$ \\
Cultural knowledge & $\begin{array}{l}\text { The nurse seeks information about other cultures and different worldviews, and how these views impact } \\
\text { a patient's health. }\end{array}$ \\
Cultural skills & $\begin{array}{l}\text { The nurse collects relevant cultural data regarding the patient's presenting problem and accurately } \\
\text { performs a culturally based physical assessment. }\end{array}$ \\
Cultural encounters & $\begin{array}{l}\text { The nurse is involved in face-to-face encounters with patients from diverse cultures. Directly interacting } \\
\text { with clients from diverse cultural groups will refine or modify a nurse's existing beliefs about a cultural } \\
\text { group and will prevent possible stereotyping that may have occurred. }\end{array}$
\end{tabular}

The IAPCC-R tool has 25 items and uses a four-point Likert scale, measuring five cultural constructs (Table 1). In the original tool, five of the items (numbers 1, 3, 11, 17 and 21) were inverted, but these items were reworded to ensure all scoring was in the same direction for the current study. The levels of cultural competence were calculated for the different constructs and the overall tool. The results can range from 25 to 100 points, with a higher score correlating with a higher level of cultural competence. In addition, the score was classified as culturally incompetent ( 25 - 50 points), culturally aware (51 - 74 points), culturally competent (75 - 90 points) and culturally proficient (91 100 points). All data were analysed using IBM SPSS version 21.0 software (IBM SPSS Inc, USA) and significance was set as $p<0.05$. Incomplete surveys were addressed in two ways: participant ratings were dropped from the analysis if $\geq 20 \%$ of the questions (five questions) were not answered $(n=1)$; and for survey instruments that had $<20 \%$ of the responses missing $(n=20)$, the missing data were imputed using IBM SPSS's mean series. Competency scores were calculated for the $104 \mathrm{CCNs}$ and compared with the demographic variables, and a Chronbach's a value was calculated for the total tool and for the individual constructs.

Ethical clearance was obtained from the University of KwaZulu-Natal ethics committee. Permission to conduct the study was also obtained from the relevant authorities. Participants were briefed about the purpose and expected procedure of the study. Verbal informed consent was obtained. Participants were informed that the study was voluntary and assured of anonymity throughout and after the study. Permission was received for the use of the instrument.

Table 2. Cultural competence score and reliability of constructs

\begin{tabular}{lll}
\hline & Mean (SD) & Chronbach's a \\
\hline Cultural awareness & $14.4(1.8)$ & 0.455 \\
Cultural desire & $14.6(1.8)$ & 0.322 \\
Cultural encounters & $12.6(2.8)$ & 0.671 \\
Cultural knowledge & $14.8(2.0)$ & 0.304 \\
Cultural skills & $13.7(2.0)$ & 0.412 \\
Cultural competence & $70.2(7.2)$ & 0.780 \\
SD = standard deviation. & &
\end{tabular}

\section{Results Respondent demographics}

A total of $105 \mathrm{CCNs}$ (response rate of $62.5 \%$ ) from eight CCUs completed the questionnaire. One respondent failed to complete any of the survey and was excluded, leaving a usable response of 104 (61.9\%). The mean age was 40.3 (standard deviation (SD) 7.9) years (range 25 - 56). Most of the respondents were female $(89,85.6 \%)$ compared with $15(14.4 \%)$ males. Over two-thirds of the respondents were black (74, 71.2\%), followed by 23 (22.1\%) Indian, 4 (3.9\%) white, and 3 (2.9\%) coloured respondents, resulting in nearly three-quarters $(74$, $71.2 \%$ ) of the respondents reporting that they spoke a language other than English at home. Most of the respondents were not university graduates, with 87 (83.7\%) reporting that they had completed a diploma, $13(12.5 \%)$ a Bachelor degree and $3(2.9 \%)$ a Masters qualification. Nearly two-thirds of the respondents were born in an urban setting $(66,63.5 \%)$.

\section{Overall cultural competence}

The overall IAPCC-R tool had reasonable internal consistency with the calculated Chronbach's a (total tool) of 0.780 , and individual constructs ranged from poor ( 0.304 for cultural knowledge) to moderate (0.671 for cultural encounters) (Table 2).

The overall cultural competence score was 70.2 (SD 7.2) out of a possible 100, with $77(74 \%)$ of the respondents scoring in the awareness range, 26 (25\%) in the competent range, and only 1 in the proficient range. The highest scores were obtained for cultural knowledge (14.8), cultural desire (14.6) and cultural awareness (14.4) (Table 2).

\section{Differences in cultural competence by demographic variables}

No significant differences for cultural competence were found for age, gender, qualification, marital status, intensive care unit type or rurality at birth. However, significant differences were found for: religion $(K=7.6$, $p=0.022$ ), with nurses reporting Christianity as their religion scoring higher than other religions (71.0 (SD 7.1) v. $64.5(6.3)$ and 65.0 (6.3)); language ( $K=14.0, p=0.001)$, specifically whether they spoke English at home v. nonEnglish speaking at home (66.3 (6.0) v. 71.8 (7.1), respectively) $(U=3.7, p<0.001)$; and race ( $K=20, p<0.001)$, with black nurses scoring significantly higher (72.2 (6.9)) compared with 
Table 3. Cultural competence by language background

\begin{tabular}{lllll}
\hline & English speaking, mean (SD) & Non-English speaking, mean (SD) & Mann-Whitney U-test & $\boldsymbol{p}$-value \\
\hline Cultural awareness & $13.8(1.6)$ & $14.6(1.8)$ & 2.4 & $0.016^{*}$ \\
Cultural desire & $14.1(1.5)$ & $14.8(1.8)$ & 2.3 & $0.021^{*}$ \\
Cultural encounters & $10.7(2.2)$ & $13.9(2.1)$ & 4.7 & $<0.001^{*}$ \\
Cultural knowledge & $14.4(1.6)$ & $15.0(1.8)$ & 1.9 & 0.055 \\
Cultural skills & $13.3(1.8)$ & $13.9(2.1)$ & 1.7 & 0.090 \\
Cultural competence & $66.3(6.0)$ & $71.8(7.1)$ & 3.7 & $<0.001^{*}$ \\
SD $=$ standard deviation. & & & & \\
*Significant at $p<0.05$. & & & &
\end{tabular}

the other races $(64.1-66.0)$. Analysing the data by English-speaking background, the higher cultural competence score for non-Englishspeaking nurses (primarily isiZulu-speaking) was driven by significant differences in cultural encounters $(p<0.001)$, cultural desire $(p=0.021)$ and cultural awareness $(p=0.016)$ (Table 3 ).

Similarly, out of the 25 items in the IAPCC-R, seven items had significant rating differences between English speaking at home v. nonEnglish speaking at home (Table 4). These items were mostly around the knowledge, skills and involvement in culture/ethnic groups outside the healthcare setting role.

\section{Discussion}

One of the key findings of this study was that the majority (74\%) of CCNs were culturally aware, but according to the tool are not yet competent. According to Campinha-Bacote ${ }^{[4]}$ cultural awareness relates to nurses becoming sensitive to the values, beliefs, lifestyles and practices of the patient, and identifying their own values, biases and prejudices. A basic characteristic of cultural competence is cultural awareness, where there is acceptance, respect and valuing of differences. Purnell and Paulanka ${ }^{[17]}$ described cultural awareness as the first step in the process of developing cultural competence.

Of the total sample, $26 \%$ were rated as competent, highlighting that more than a quarter of the CCNs were involved in culturally appropriate healthcare interventions and interactions. According to Starr and Wallace, ${ }^{[18]}$ being culturally competent allows nurses to engage in practice, policy and advocacy activities that address health disparities more effectively. The ultimate goal of cultural competence is to make healthcare more responsive to the needs of patients, and increase their satisfaction with and access to healthcare, decrease inappropriate differences in the characteristics and quality of care provided, and close the gaps in health status in diverse populations. ${ }^{[19]}$

A second finding of the study was that although there were concerns about the internal consistency of the tool with respect to measuring the constructs, the findings also highlighted the differences between the different constructs of cultural competence. Of the five constructs, cultural knowledge scored the highest among both English- and nonEnglish-speaking CCNs. This indicated that CCNs were most involved in the process of making an effort to learn about increasing and improving their own cultural knowledge, focusing on health-related beliefs and cultural values, disease conditions and treatment options. This is similar to the findings of Sealey et al., ${ }^{[20]}$ who also reported that strategies for developing cultural knowledge towards cultural competence should be a priority. These authors further suggested that staff should be encouraged to attend continuing education programmes on cultural competence to improve their knowledge in this area. This continuing education, coupled with more local and/or international cross-cultural encounters, should significantly improve the overall cultural competence of the staff.

Cultural desire was rated the second highest among the constructs. It was also evident that CCNs were motivated towards engaging in the process of becoming culturally competent. The concepts of caring and love are central to the construct of cultural desire and the capacity to sacrifice. The findings highlighted that CCNs were willing to provide care that is culturally responsive. As reflected by Campinha-Bacote ${ }_{1}^{[4]}$ the constructs of cultural awareness, knowledge, skills, encounters and desire have an interdependent relationship with each other, and no matter where the healthcare provider enters into the process of cultural competence, all five constructs must be addressed or experienced.

The lowest scoring constructs were cultural skill and encounters. The low rating for cultural skill may highlight that CCNs were not as involved in collecting relevant cultural data regarding the patients' presenting problem as they were at accurately performing a culturally based physical assessment. This is most likely related to the fact that CCNs were not competent in engaging in such an assessment. According to Campinha-Bacote ${ }_{1}^{[4]}$ performing a cultural assessment requires skills in order to obtain accurate information from the patient so as to formulate a mutually acceptable and relevant treatment plan for each patient problem. Although there are numerous international cultural assessment tools, currently there are limited assessment tools within the SA health context. According to Zwane and Poggenpoel, ${ }^{[2]]}$ nursing should include cultural content within undergraduate training in SA in order to provide culturally appropriate care to patients. According to Barbee and Gibson: ${ }^{[2]}$ 'We need to recognise that talking and writing about cultural diversity without consciously and forthrightly dealing with [it] in nursing education [and practice] are essentially empty exercises that would continue to perpetuate the status quo.'

The construct that scored the lowest was cultural encounters. The scores were low for both English- and non-English-speaking respondents. This highlights that CCNs are less likely to be involved in face-to-face encounters with patients from diverse cultures. A possible reason for this within the critical care context is that most patients are often ventilated and sedated, and when CCNs do interact on a faceto-face basis with a patient, the CCNs find this interaction stressful. According to Cang-Wong et al. ${ }^{[23]}$ nurses often experience workplace stressors when they are unable to deal with cultural differences. Even though SA is seen as the 'rainbow nation' and a culturally diverse group, most cultural/ethnic groups still have limited interaction with other groups on a social level. People may find interaction with their 
own cultural group more comfortable than interacting with a different group. Another possible reason for this low score could be that even though we are seen as a diverse nation, we still need to learn about other cultural/ethnic groups; in the meantime, the limited knowledge and awareness limits our encounters.

A third finding from the study was the reported differences in self-rating of cultural competence by English- and non-Englishspeaking nurses. From the findings of the study, it was evident that non-English-speaking CCNs rated themselves higher on all of the individual constructs than English-speaking CCNs. This indicated that CCNs who are non-English speaking indicated a higher degree of cultural awareness towards cultural competence than Englishspeaking CCNs. It can be deduced that English-speaking CCNs found it more challenging to provide culturally congruent care than their colleagues. A possible reason for this is that patients admitted to this tertiary hospital are largely non-English speaking. According to Cang-Wong et al., ${ }^{[23]}$ communication is the central factor in providing transcultural care and is one of the most significant challenges when a nurse and patient do not speak the same language. This highlights the importance for healthcare workers to integrate culture and language in order to improve health outcomes for patients from diverse cultural backgrounds.

\section{Study limitations}

Study limitations included the purposive sampling design and the use of only one hospital. In addition to this, the tool reflected weakness in terms of low consistency in measuring the constructs, although the overall tool was consistent.

\section{Conclusion}

The IAPCC-R tool had reasonable internal consistency, although individual constructs ranged from poor (for cultural knowledge) to moderate (for encounters). This study has reinforced the need to examine the reliability and validity of this instrument in different contexts. The IAPCC-R tool is linked to a particular theoretical framework, making it less useful for use as a generic tool in measuring cultural competence. There are many instruments available to assess cultural competence, and despite the limitations associated with these tools, there is still value in the initial assessment of cultural competence they provide. Cultural competence is a challenge to assess and will continue to be so for researchers.

References

Cowan D, Norman I. Cultural competence in Nursing: New meanings. J Transcult Nurs 2006;17(1):82-88. [http://dx.doi.org/10.1177/1043659605281976]

2. College of Nurses of Ontario. Standard of Care. 2010. Culturally sensitive care. Practice Guidelines http://www.cno.org/Global/docs/prac/41040_CulturallySens.pdf (accessed 20 November 2013).

3. Williamson $M$, Harrison L. Providing culturally appropriate care: A literature review. Int J Nurs Stud 2010;47(6):761-769. [http://dx.doi.org/10.1016/j.jijnurstu.2009.12.012]

4. Campinha-Bacote J. The process of cultural competence in the delivery of healthcare: A mode of care. J Transcult Nurse 2002;13(3):181-184.

5. Papadopoulos I. Transcultural Health and Social Care: Development of Culturally Competent Practitioners. Edinburgh: Elsevier Health Sciences, 2006.

6. Vanderpool HK. Report of the ASHP Ad Hoc Committee on Ethnic Diversity and Cultural Competence. Am J Health Syst Pharm 2005;62(18):1924-1930. [http://dx.doi.org/10.2146/ajhp050100]

7. Seright T. Perspectives of registered nurse cultural competence in a rural state - Part 11. Online Journal of Rural Nursing and Healthcare 2007;7(1):57-69.

8. Jirwe M, Gerrish K, Keeney S, Emami A. Identifying the core components of cultural competence: Findings from a Delphi study. J Clin Nurs 2009;18(18):2622-2634. [http://dx.doi. org/10.1111/j.1365-2702.2008.02734.x]

9. Andrews MM, Boyle JS. Transcultural Concepts in Nursing Care. Philadelphia: Lippincott, 2003.

10. Wittig DR. Knowledge, skills and attitudes of nursing students regarding culturally congruent care of Native Americans. J Transcult Nurs 2004:15(1):54-61. [http://dx.do org/10.1177/1043659603260036]

11. Ballard R. The implications of cultural diversity for healthcare practice: An anthropological perspective. http://archiv.ub.uni-heidelberg.de/savifadok/247/1/healthcare_1.pdf (accessed 25 November 2013).

12. Flowers DL. Cultural competent nursing care, a challenge for the 21st century. Crit Care Nurs 2004;24(4):48-52.

13. Leininger MM, McFarland M. Culture Care in an Urban Nursing Home with African and AngloAmericans. Detroit: Wayne State University, 1991.

14. Leininger MM. Culture Care Diversity and Universality: A Theory of Nursing. New York: National League for Nursing Press, 1991.

15. Albarran J, Rosser E, Bach S, Uhrenfeldt L, Lundberg P, Law K. Exploring the development of a cultural care framework for European caring science. Int $J$ Qual Stud Health Well-being of a cultural care framework for European caring science. Int
2011;6(4):11457. [http://dx.doi.org/10.3402/qhw.v6i4.11457]

16. Olt $H$, Jirwe $M$, Gustavsson $P$, Emami A. Psychometric evaluation of the Swedish adaptation of the Inventory for Assessing the Process of Cultural Competence Among Healthcar Professionals, Revised (IAPCC-R) T Transcult Nurs 2010:21(1):55-64. [http://dx.do. org/10.1177/1043659609349064

17. Purnell L, Paulanka B. Transcultural Healthcare: A Culturally Competent Approach. Philadelphia: FA Davis, 2008.

18. Starr S, Wallace DC. Self-reported cultural competence of public health nurses in a Southeastern US Public Health Department. Public Health Nurs 2009;26(1):48-57. [http://dx.doi.org/10.1111/ j.1525-1446.2008.00753.x]

19. Anderson LM, Scrimshaw SC, Fullilove MT, Fielding JE, Normand J. Culturally competent healthcare systems. A systematic review. Am J Prev Med 2003;24(3 Suppl):69-79.

20. Sealey LJ, Burnett M, Johnson G. Cultural competence of baccalaureate nursing faculty: Are we up to the task? J Cult Divers 2006;13(3):131-140.

21. Zwane $S$, Poggenpoel $M$. Student nurses experiences of interacting with culturally diverse psychiatric patients. Curationis 2000;23(2):25-31.

22. Barbee EL, Gibson SE. Our dismal progress: The recruitment of non-whites into nursing. J Nurs Educ 2001;40(6):243-244.

23. Cang-Wong C, Murphy SO, Adelman T. Nursing responses to transcultural encounters: What nurses draw on when faced with a patient from another? Perm J 2009;13(3):31-36. 